\title{
Resiquimod Topical Gel
}

National Cancer Institute

\section{Source}

National Cancer Institute. Resiquimod Topical Gel. NCI Thesaurus. Code C88259.

A topical gel containing the Toll-like receptor (TLR) agonist resiquimod, an imidazoquinolinamine and with potential immunomodulating activity. Resiquimod binds toT LR7 and 8, mainly on dendritic cells, macrophages, and B-lymphocytes, and activates the TLR signaling pathway, resulting in the induction of the nuclear translocation of transcription activator NF-kB and activation of other transcription factors; subsequently, gene expression increases and the production of cytokines increases, especially interferon-alpha (INF-a), resulting in the enhancement of T-helper 1 (Th1) immune responses. In addition, topical application of resiquimod appears to activate epidermal Langerhans cells, leading to an enhanced activation of T-lymphocytes. 